\title{
Editorial
}

\section{Multiple Perspectives on Interiority}

Paramita Atmodiwirjo

Universitas Indonesia

Indonesia

There has been a growing discourse on interiority in designrelated discipline, which attempts to extend our understanding of inhabitation in space. The idea of interiority refers to the internal aspects that make and condition the interior. It poses challenges to the way we see the interior, which is not bounded to the internality of architectural space and is not limited to the physical enclosure of the environment (McCarthy, 2005). From the perspective of the individual, Merleau-Ponty explains interiority as pertaining to the inner and psychological life as opposed to exteriority that refers to the material world (Olkowsky \& Morley, 1999). The idea of interiority acknowledges the subjectivity in the process of inhabitation, however, it is not entirely free from its materiality. Interiority might be explained and experienced through the sensorial encounter, personal engagement, and social interaction, in which tangible and intangible aspects of the interior may be entangled.

The idea of interiority provokes further exploration of interior in its multiplicity and complexity. Interiority might be understood as a range of internal rules, logics, systems or mechanisms explain how interior works (such as in Eisenman, 1999; Rahim, 2010). Interiority might be understood from the perspectives of different actors engaged in the process of inhabitation, in which subjectivity and personality may take place side by side with the materiality of inhabitation. Interiority should be comprehended within its specific socio-cultural contexts, which might trigger particular ideas of interiority. The idea of interiority becomes relevant to address various contexts of interior inhabitation, which may range from domestic to urban interior, from personal to collective interior, from historic to contemporary interior, and from indigenous to global interior. 
Thewiderange of interior contexts offers rich and unique perspectives to exercise the idea of interiority, as well as possibilities to explore and define creative intervention practice. The understanding of interiority should also become a vehicle to respond to various critical issues of human inhabitation. It is necessary to explore how the knowledge on interiority could contribute to in achieving the wellbeing of human life, through the design research and intervention that is aimed at better interior and architectural environment.

The initiative to establish this journal as a medium for dialog to discuss the idea of interiority has began since the first [in]arch Conference on Interiority and Interior Architecture at Universitas Indonesia in 2014, where scholars from various design-related disciplines from different regions of the world gathered and established a dialog on various topics related to interiority. The conference became an opportunity to reinterpret and extend the knowledge on interiority, along with the development of the methods and approaches of interior research, practice and pedagogy. The dialogue during the conference suggested possibilities to further extend the discourse on interiority, which is then realised through the publication of this journal.

The first issue of this journal includes five papers and one review article that represent a range of perspectives on interiority. They contribute to the dialogue on interiority that addresses questions and issues in different interior contexts. Mark Pimlott highlights the condition of the interior as a critical aspect of understanding the individual experience of interiority within the urban context. Edward Hollis explores the narrative of interiors through three vignettes telling the stories of interior. They suggest the idea of the interior as a sort of performance, and the way we speak of interior becomes the way interior exists. John Stanislav Sadar challenges the materiality in the interior by exploring the potential of quasimaterials, such as light, sound, temperature, and humidity, that are present beyond the common physical elements of the interior.

The other two papers address the questions and issues of interiority in particular interior contexts. Christina Deluchi illustrates the interior context in the city of Medellin, Columbia, where its urban interiority is characterised by the regulation, protection, and surveillance. Ayu Suci Warakanyaka and Yandi Andri Yatmo trace the layers of surfaces that are present in the old buildings in Semarang, Central Java, and examine these layers as media to reveal the inhabitation of space through time. Finally, Mikhael Johanes and Arif Rahman Wahid reviewed an exhibition on indigenous materiality, which celebrates the local materials in various regions in Indonesia as reflections of social and cultural values of the society. The exhibition highlights the 
importance of the processes and the actors behind the materiality of interior and architecture.

By extending the discourse on interiority, this journal challenges the emergence of various perspectives in defining and developing further design research agenda and promotes multidisciplinary dialogue in exploring the idea of interiority. It also hopefully becomes the trigger for extending the practice of design - architecture, interior design, spatial design and other relevant design fields - to address more appropriately the social, cultural and behavioural aspects of the space inhabitation.

\section{References}

Eisenman, P. (1999). Diagram diaries. London: Thames and Hudson.

McCarthy, C. (2005). Toward a definition of interiority. Space and Culture, 8(2), 112-125.

Olkowsky, D. \& Morley, J. (Eds.). (1999). Merleau-Ponty, Interiority and exteriority, psychic life and the world. Albany, NY : State University of New York Press.

Rahim, A. (2010). Interiorities. Architectural Design, 80(2), 24-31. 